\title{
Excitation of Triple Giant Resonances in Heavy-Ion Reactions*
}

\author{
M.S. Hussein ${ }^{1}$, B.V. Carlson ${ }^{2}$, L.F. Canto ${ }^{3}$, and A.F.R. de Toledo Piza ${ }^{1}$ \\ ${ }^{1}$ Instituto de Física, Universidade de São Paulo, \\ CP 66318, 05315-970 São Paulo, Brazil \\ ${ }^{2}$ Departamento de Física, Instituto Tecnológico de Aeronáutica - CTA \\ 12228-900 São José dos Campos, SP, Brazil \\ ${ }^{3}$ Instituto de Física, Universidade do Rio de Janeiro, \\ CP 68528, 21945-970 Rio de Janeiro RJ, Brazil
}

(November 3, 2018)

\begin{abstract}
We calculate the cross-section for the excitation and subsequent decay of triple giant resonances (TGDR) in several nuclei excited with heavy ions. The recently developed coherent plus incoherent theory for the excitation in conjunction with the hybrid decay model of Dias-Hussein-Adhikari are used for the purpose. It is emphasized that the direct decay of the TGR is expected to deviate appreciably from the harmonic limit especially at low bombarding energies, owing to the incoherent contribution.
\end{abstract}

PACS Numbers: 25.70.De, 24.30.Cz, 21.10.Re

*Supported in part by CNPq and FAPESP. 
The study of the double giant dipole resonance in nuclei has received a considerable amount of attention over the last 15 years [1]. Both the pion double charge exchange and relativistic heavy ion Coulomb excitation reactions have been used to probe this large amplitude collective motion in may fermion systems. The quest for the similar double plasmon resonance in metallic clusters is underway [2]. Plans are also in progress to search for the triple giant dipole resonance (TGDR) in nuclei [3]. It is clearly of importance to supply theoretical estimates of the cross-section as well as the different decay branching ratios of these exotic collective modes. This is the purpose of the present paper. We use the recently developed coherent plus incoherent excitation theory of Ref. [4] in conjunction with the hybrid decay model of Dias-Hussein-Adhikari (DHA) of Ref. [5].

The existing models for the calculation of the excitation cross-section of DGDR can be grouped into three categories: a microscopic structure model in conjunction with second order Coulomb excitation perturbation theory [6], a macroscopic, oscillator (harmonic or anharmonic) model in conjunction with coupled channels Coulomb excitation theory [7] and finally the recently developed average plus fluctuation model $[4,8]$. In this latter model the average cross-section is calculated according to the theory developed in [9], where the simple, double, etc. giant resonances are considered as doorway states belonging to the spectrum of a damped harmonic oscillator.

A fully microscopic structure calculation of the excitation cross-section of the TGDR resonance is prohibitively difficult. A huge number of three particle-three hole configurations have to be dealt with in a coupled channel context. A detailed account of the spreading of the TGDR would require the inclusion in the calculation of at least the four particle - four hole subspace. Not having available such a detailed description we opt for using our coherent + fluctuation model [8]. The TGDR excitation cross-section is found to have the form

$$
\sigma^{(3)}=\sigma_{c}^{(3)}+\sigma_{f l}^{(3)}(2)+\sigma_{f l}^{(3)}(1)
$$


where $\sigma_{c}^{(3)}$ is the average cross-section for the coherent excitation of the three phonons which proceeds through the one and two phonon states in a typical three-step description. This cross section can be guaranteed for the coupled channel Coulomb excitation model which contains explicit reference to flux loss from there states owing to their spreading into more complex configurations. The cross-section $\sigma_{f l}^{(3)}(2)$ corresponds to fluctuation contribution arising from the decay of an intermediate collective GR phonon into the complicated background followed by the excitation of another collective phonon on the background states (the Brink-Axel phonon). The final states involved in this cross-section contains two collective phonons in contrast to $\sigma_{c}^{(3)}$. Finally $\sigma_{f l}^{(3)}(1)$ contains contributions that lead to only one phonon in the final state. In terms of the time sequence of events, $\sigma_{c}^{(3)}$, being a three-step process, is the fastest, followed by the four-step process accounted for by $\sigma_{f l}^{(3)}(2)$ (this is a four-step process since besides the three excitation steps one has one internal mixing step) and finally the five step process contained in $\sigma_{f l}^{(3)}(1)$. The DGR cross section may be similarly decomposed as $\sigma^{(2)}=\sigma_{c}^{(2)}+\sigma_{f l}^{(2)}(1)$.

In Ref. [8] we obtained the following estimates for $\sigma_{f l}^{(3)}(i)$ :

$$
\begin{gathered}
\sigma_{f l}^{(3)}(2)=\left(\frac{2}{3}\right) \frac{\Gamma_{1}^{\downarrow} \tau_{c}}{\hbar} \sigma_{c}^{(3)}, \\
\sigma_{f l}^{(3)}(1)=\left(\frac{1}{3}\right)\left(\frac{\Gamma_{1}^{\downarrow} \tau_{c}}{\hbar}\right)^{2} \sigma_{c}^{(3)},
\end{gathered}
$$

where $\Gamma_{1}^{\downarrow}$ is the spreading width of the single phonon $G R$ and $\tau_{c}$ is the average collision time given by $\frac{b_{o}}{\gamma v}$, with $b_{o}$ being the grazing impact parameter, $v$ the asymptotic relative velocity and $\gamma$ is the Lorentz factor, $\gamma=\left(1-\left(\frac{v}{c}\right)^{2}\right)^{-1 / 2}$.

The above fluctuation contribution become insignificant at very high energies where the systems proceeds very quickly though the sequence ground state $\rightarrow G D R \rightarrow$ $D G D R \rightarrow T G D R$. When the bombarding energy is lowered, the fluctuation effects may become appreciable [8]. The estimates above were found to be quite reasonable 
when compared to the more elaborate model of Ref. [4] at relatively high bombarding energies. At lower energies, clearly, for the evaluation of the different contributions to the total excitation cross-section, Eq. (1), one should rely on the latter, more precise model. In order to discuss the decay of the final states into the open channels, we need to know the values of the different contributions to $\sigma^{(2)}$ and $\sigma^{(3)}$.

We have calculated the excitation cross-sections, $\sigma^{(1)}, \sigma^{(2)}$ and $\sigma^{(3)}$, for various nuclei incident on ${ }^{208} \mathrm{~Pb}$ at several bombarding energies, using a three-dimensional (3D) generalization of the model of Ref. [4]. The 3D time evolution equation used to describe the excitation and decay of the GDR phonons possesses the same form as the one-dimensional equation of Ref. [4]. However, the collective and statistical excited states of the 3D model take into account all possible combinations of the (two) transverse and (one) longitudinal degrees of freedom, which yield 3 coherent one-phonon states, 6 coherent two-phonon states and 10 coherent three-phonon states as well as a multitude of states containing a mixture of coherent and statistical excitations. Decays of the three types of phonons to the statistical background are assumed to occur independently but to each obey Bose-Einstein statistics.

The Coulomb interaction matrix elements used to describe the transverse modes of the GDR excitation in the 3D model are the physically appropriate ones, as given in Ref. [9]. The longitudinal Coulomb interaction matrix element, however, is modified from the form given there. It is reduced to a term proportional to the longitudinal component of the eletric field, in analogy to the transerse terms, but which differs from the expression given in Ref. [9] by a total time derivative. The latter term can be extracted from the equations and discarded when only the coherent excitation is included. This is no longer the case when decay to the statistical states is taken into account. Nevertheless, we have neglected its contribution here.

As in Ref. [4], the coupled equations of motion are solved as a function of impact parameter to yield asymptotic occupation probabilities. Effective asymptotic occupation probabilities are defined, for states that decay, as the sum over the probability that decays out of each state during the time evolution. Cross sections are obtained 
by integrating each probability $\mathrm{x}$ differential area over impact parameter and summing over polarizations.

The various contributions to the cross sections are easily extracted from the theoretical calculations. In Table I, we present the coherent and fluctuation contributions to the DGDR cross section, $\sigma_{c}^{(2)}$ and $\sigma_{f l}^{(2)}(1)$ for various nuclei incident on ${ }^{208} \mathrm{~Pb}$ at several energies. In Table II, we present the contributions to the TGDR cross section, $\sigma_{c}^{(3)}, \sigma_{f l}^{(3)}(2)$, and $\sigma_{f l}^{(3)}(1)$. We observe that the cross sections increase dramatically with the charge of the projectile. As is well known, the coherent two-phonon cross sections scales approximately as the charge Z squared, while the three-phonon one scales as $Z^{3}$. We also observe that the coherent contribution to the cross sections only dominates at relatively high incident energies. At the lowest energy shown, $10 \mathrm{MeV} /$ nucleon, the cross sections involving statistical phonons are substantially larger than the coherent ones. At $E / A=100 \mathrm{MeV}$, it is clear from the tables that the fluctuation contribution to the DGDR cross section is about as large as the coherent one, while the fluctuation contribution to the TGDR is about three times larger than the coherent contribution.

We turn now to the decay of the DGR and TGR. We first remind the reader of the hybrid direct+fluctuation decay model of DHA [5]. According to this model, which has been extensively used in the analysis of the decay data $[10,11]$, the GR decays to a find channel $f$ in the following manner:

$$
\begin{aligned}
\sigma_{f}^{(1)} & =\sigma^{(1)}\left[\left(1-\mu_{1}\right) \frac{\tau_{f}^{(G R)}}{\sum_{j} \tau_{j}^{(G R)}}+\mu_{1} \frac{\tau_{f}^{(C N)}+\mu_{1} \tau_{f}^{(G R)}}{\sum_{j}\left(\tau_{j}^{(C N)}+\mu_{1} \tau_{j}^{(G R)}\right)}\right] \\
& \equiv \sigma^{(1)}\left(P_{f}^{\uparrow}+P_{f}^{\downarrow}\right)
\end{aligned}
$$

where $\sigma^{(1)}$ is the one phonon excitation cross section discussed before, while $\mu_{1}=\frac{\Gamma_{1}^{\downarrow}}{\Gamma_{1}}$ and the $\tau^{\prime} s$ are the appropriate transmission coefficients. We have written the probability of populating the final channel $f$ through direct decay of the GR as

$$
P_{f}^{\uparrow}=\left(1-\mu_{1}\right) \frac{\tau_{f}^{(G R)}}{\sum_{j} \tau_{j}^{(G R)}},
$$


and the probability of of populating the channel $f$ through the statistical states as

$$
P_{f}^{\downarrow}=\mu_{1} \frac{\tau_{f}^{(C N)}+\mu_{1} \tau_{f}^{(G R)}}{\sum_{j}\left(\tau_{j}^{(C N)}+\mu_{1} \tau_{j}^{(G R)}\right)}
$$

Note that the statistical decay component contains explicit reference to the GR direct transmission, $\left(\mu_{1} \tau_{f}^{(G R)}\right)$.

Before entering into the details of the decay of the multiple giant resonances, let us first analyze the decomposition into direct decay and decay into the statistical states. For this purpose, we use the branching ratios

$$
P^{\uparrow}=\sum_{f} P_{f}^{\uparrow}=1-\mu_{1} \quad \text { and } \quad P^{\downarrow}=\sum_{f} P_{f}^{\downarrow}=\mu_{1} .
$$

The decomposition of the single GR decay is a direct result of Eq. (四),

$$
\sigma^{(1)} \rightarrow\left(P^{\uparrow}+P^{\downarrow}\right) \sigma^{(1)}
$$

To decompose the decay of the multiple giant resonances into direct and statistical parts, we assume that each of the collective phonons decays independently. The decay of the coherent contributions to the DGR and TGR can then be decomposed as

$$
\begin{aligned}
\sigma_{c}^{(2)} & \rightarrow\left(P^{\uparrow}+P^{\downarrow}\right)^{2} \sigma_{c}^{(2)} \\
& =\left(P^{\uparrow 2}+2 P^{\uparrow} P^{\downarrow}+P^{\downarrow 2}\right) \sigma_{c}^{(2)},
\end{aligned}
$$

and

$$
\begin{aligned}
\sigma_{c}^{(3)} & \rightarrow\left(P^{\uparrow}+P^{\downarrow}\right)^{3} \sigma_{c}^{(3)} \\
& =\left(P^{\uparrow 3}+3 P^{\uparrow 2} P^{\downarrow}+3 P^{\uparrow} P^{\downarrow 2}+P^{\downarrow 3}\right) \sigma_{c}^{(3)} .
\end{aligned}
$$

That is, the coherent contribution to the DGR can decay through direct decay of each of the collective phonons, through a direct decay of one of the colective phonons and decay into the statistical states of the other, or through decay into the statistical states of both of the phonons. Decay of the coherent contribution to the TGR takes into account the different possible direct or statistical decays of the three initial phonons. 
We can analyze the decomposition of the decay of the fluctuating contributions to the DGR and TGR cross sections in a similar manner. We need only take into account the number of collective phonons in each of the contributions. Thus, the decay of the fluctuating component of the DGR can be decomposed as

$$
\sigma_{f l}^{(2)}(1) \rightarrow\left(P^{\uparrow}+P^{\downarrow}\right) \sigma_{f l}^{(2)}(1)
$$

while the decay of the fluctuating components of the TGR can be decomposed as

$$
\sigma_{f l}^{(3)}(2) \rightarrow\left(P^{\uparrow 2}+2 P^{\uparrow} P^{\downarrow}+P^{\downarrow 2}\right) \sigma_{f l}^{(3)}(2),
$$

and

$$
\sigma_{f l}^{(3)}(1) \rightarrow\left(P^{\uparrow}+P^{\downarrow}\right) \sigma_{f l}^{(3)}(1)
$$

We can now combine the various terms into decompositions of the decay of the total DGR and TGR cross sections,

$$
\begin{aligned}
\sigma^{(2)} & \rightarrow P^{\uparrow 2} \sigma_{c}^{(2)} \\
& +P^{\uparrow}\left(2 P^{\downarrow} \sigma_{c}^{(2)}+\sigma_{f l}^{(2)}(1)\right) \\
& +P^{\downarrow}\left(P^{\downarrow} \sigma_{c}^{(2)}+\sigma_{f l}^{(2)}(1)\right),
\end{aligned}
$$

and

$$
\begin{aligned}
\sigma^{(3)} & \rightarrow P^{\uparrow 3} \sigma_{c}^{(3)} \\
& +P^{\uparrow 2}\left(3 P^{\downarrow} \sigma_{c}^{(3)}+\sigma_{f l}^{(3)}(2)\right) \\
& +P^{\uparrow}\left(3 P^{\downarrow} 2 \sigma_{c}^{(3)}+2 P^{\downarrow} \sigma_{f l}^{(3)}(2)+\sigma_{f l}^{(3)}(1)\right) \\
& +P^{\downarrow}\left(P^{\downarrow 2} \sigma_{c}^{(3)}+P^{\downarrow} \sigma_{f l}^{(3)}(2)+\sigma_{f l}^{(3)}(1)\right),
\end{aligned}
$$

where we have collected terms according to the number of direct decays involved.

In medium to heavy nuclei, one expects the spreading to dominate over the escape from the GR, which implies $P^{\downarrow}=\mu_{1} \simeq 1$. In this case, we see that the completely statistical DGR and TGR decay cross sections, given by the last term in the preceding 
two equations, will be approximately proportional to their total excitation cross sections. They will not distinguish between the coherent and fluctuating components of the cross sections. Further analysis of the decay of the statistical component to equilibrium can be quite complicated. Usually, however, particle emission from the statistical component can be well described using the equilibrium Hauser-Feshbach formalism.

To best view the distinction between the coherent and fluctuation contributions to the GDR cross sections, we look for effects in the direct decay from the giant resonance. We have already analyzed the effects of the coherent and fluctuating components on exclusive decays of the DGDR. [12]. Here, we wish to analyze their effects on inclusive decay cross sections of the DGR and TGR.

To separate direct contributions to an inclusive cross section from the statistical contributions, it is necessary to concentrate one's attention on the high-energy end of the emission spectrum. There, the statistical weight of the high energy residuals in the statistical cross section strongly suppresses emission, leaving the direct emission to dominate the spectrum. To obtain the inclusive emission cross section from our decomposition of the decay of the excitation cross sections through direct and statistical modes, Eqs. 14 and 15, we take into account that each of the direct decay factors $P^{\uparrow}$ can contribute independently to direct emission in channel $f$ with a relative probability $P_{f}^{\uparrow} / P^{\uparrow}$. Thus, for example, each of the factors of $P^{\uparrow}$ in the $P^{\uparrow 2}$ (first) term of Eq. 14 contributes to the direct emission in channel $f$ with probability $P_{f}^{\uparrow} / P^{\uparrow}$, resulting in a total contribution of $2 P_{f}^{\uparrow} / P^{\uparrow}$ of the term to the emission cross section. In general, we include a factor of $P_{f}^{\uparrow} / P^{\uparrow}$ for each of the factors of $P^{\uparrow}$ appearing in the equations. We neglect the terms containing only statistical decays, since we have $P_{f}^{\downarrow} / P^{\downarrow} \approx 0$ at the high end of the emission spectrum.

We point out that the relative decay probabilities $P_{f}^{\uparrow} / P^{\uparrow}$ are not quit the same for each of the decays, since conservation laws constrain the energies and angular momenta of the emitted particles to values consistent with the residual values in the nucleus. The residual nuclear values will not be identical for a cold nucleus (first emission) and a hot one (second and later emissions). Nevertheless, the general structure of the phonon, 
which we assume to be essentially a one particle-one hole state, assure that the value of $P_{f}^{\uparrow} / P^{\uparrow}$ will be about the same for each of the emissions.

Using the rules above for estimating the contribution of the $P^{\uparrow}$ factors to emission, we find the direct inclusive emission spectra to be given by

$$
\begin{aligned}
\sigma_{f}^{(2)} & =2 \frac{P_{f}^{\uparrow}}{P^{\uparrow}} P^{\uparrow 2} \sigma_{c}^{(2)} \\
& +\frac{P_{f}^{\uparrow}}{P^{\uparrow}} P^{\uparrow}\left(2 P^{\downarrow} \sigma_{c}^{(2)}+\sigma_{f l}^{(2)}(1)\right),
\end{aligned}
$$

and

$$
\begin{aligned}
\sigma_{f}^{(3)} & =3 \frac{P_{f}^{\uparrow}}{P^{\uparrow}} P^{\uparrow 3} \sigma_{c}^{(3)} \\
& +2 \frac{P_{f}^{\uparrow}}{P^{\uparrow}} P^{\uparrow 2}\left(3 P^{\downarrow} \sigma_{c}^{(3)}+\sigma_{f l}^{(3)}(2)\right) \\
& +\frac{P_{f}^{\uparrow}}{P^{\uparrow}} P^{\uparrow}\left(3 P^{\downarrow 2} \sigma_{c}^{(3)}+2 P^{\downarrow} \sigma_{f l}^{(3)}(2)+\sigma_{f l}^{(3)}(1)\right),
\end{aligned}
$$

which reduce to

$$
\sigma_{f}^{(2)}=P_{f}^{\uparrow}\left(2 \sigma_{c}^{(2)}+\sigma_{f l}^{(2)}(1)\right)
$$

and

$$
\sigma_{f}^{(3)}=P_{f}^{\uparrow}\left(3 \sigma_{c}^{(3)}+2 \sigma_{f l}^{(3)}(2)+\sigma_{f l}^{(3)}(1)\right) .
$$

Here we see clearly the importance of the (more) coherent contributions to the direct emission spectra. Each of the excitation cross sections contributes according to the number of collective phonons it possesses.

We can similarly analyze the contribution to the inclusive cross sections of each of the components of the of the excitation cross sections. In particular, we determine the contribution of the coherent DGR and TGR to the emission spectra to be

$$
\sigma_{c, f}^{(2)}=2 P_{f}^{\uparrow} \sigma_{c}^{(2)} \quad \text { and } \quad \sigma_{c, f}^{(3)}=3 P_{f}^{\uparrow} \sigma_{c}^{(3)} .
$$

We could call these values the harmonic limit of the cross section. Comparing these to the values for the total direct emission, we find

$$
\frac{\sigma_{f}^{(2)}}{\sigma_{c, f}^{(2)}}=1+\frac{1}{2} \frac{\sigma_{f l}^{(2)}(1)}{\sigma_{c}^{(2)}},
$$


and

$$
\frac{\sigma_{f}^{(3)}}{\sigma_{c, f}^{(3)}}=1+\frac{2}{3} \frac{\sigma_{f l}^{(3)}(2)}{\sigma_{c}^{(3)}}+\frac{1}{3} \frac{\sigma_{f l}^{(3)}(1)}{\sigma_{c}^{(3)}}
$$

Thus, a considerably larger direct decay may occur if the fluctuation contributions are important, which may occur at lower bombarding energies. Of course, one could obtain deviation of the direct decay from the harmonic limit (two or three independently decaying phonons), if anharmonic effects were allowed, This, however, will imply deviation of the spectrum of the oscillator from the harmonic sequence, which seems to be borne out neither by experiment [1] nor by calculation [7].

In conclusion, we have, in this paper, calculated the excitation cross-section and studied the the decay properties of the double and triple giant dipole resonances of various nuclei as excited in Coulomb collisions with ${ }^{208} \mathrm{~Pb}$. It was found that the degree of deviation of the direct decay from the limit of two or three independently decaying collective phonons depends significantly on the bombarding energy, $E$, and can be appreciable at low values of $E$. 


\section{REFERENCES}

[1] See, e.g., T. Aumann, P.F. Bortignon and H. Emling, Ann. Rev. Nucl. Part. Sci. 48, 351 (1998).

[2] B.V. Issendorff, Talk given at DGDR99 ECT* - Trento, Italy, May 1999.

[3] N. Frascaria, Talk given at DGDR99 ECT* - Trento, Italy, May 1999.

[4] B.V. Carlson, M.S. Hussein, A.F.R. de Toledo Piza and L.F. Canto, Phys. Rev. C60, 014604 (1999); B.V. Carlson and M.S. Hussein, Phys. Rev. C59, R2343 (1999).

[5] H. Dias, M.S. Hussein and S.K. Adhikari, Phys. Rev. Lett 57, 1998 (1986); R. Bonetti, M. Chadwick, P. Hodgson, B.V. Carlson and M.S. Hussein, Phys. Rep. 202, 171 (1991).

[6] V. Yu Ponomarev, E. Vigezzi, P.F. Bortignon, R.A. Broglia, G. Colo, G. Lazzari, V.V. Voronov and G. Baur, Phys. Rev. Lett. 72, 1168 (1994).

[7] See, e.g., C.A. Bertulani and V. Yu Ponomarev, Phys. Rep. 321139 (1999).

[8] B.V. Carlson, L.F. Canto, S. Cruz-Barrios, M.S. Hussein and A.F.R. de Toledo Piza, Ann. Phys. (New York) 276111 (1999); L.F. Canto, B.V. Carlson, M.S. Hussein and A.F.R. de Toledo Piza, Phys. Rev. C60, 064624 (1999).

[9] L.F. Canto, A. Romanelli, M.S. Hussein and A.F.R. de Toledo Piza, Phys. Rev. Lett. 72, 2147 (1994); C.A. Bertulani, L.F. Canto, M.S. Hussein and A.F.R. de Toledo Piza, Phys. Rev. C53, 334 (1996).

[10] A. Bracco, J.R. Beene, N. Van Giai, P.F. Bortignon, F. Zardi and R.A. Broglia, Phys. Rev. Lett. 60, 2603 (1988); A. Bracco, P.F. Bortignon and R.A. Broglia, "Giant Resonances", 1996.

[11] C.A.P. Ceneviva, N. Teruya, H. Dias and M.S. Hussein, Phys. Rev. C57, 3220 (1998); ibid C55, (1997). 
[12] B.V. Carlson, M.S. Hussein, and A.F.R. de Toledo Piza, Phys. Lett. 431B, 249 (1998). 


\section{TABLES}

TABLE I. Contributions of the coherent and fluctuation components to the DGDR excitation cross section (in mb) of various projectiles incident on a lead target at several different values of the incident energy.

\begin{tabular}{|c|c|c|c|c|c|c|}
\hline \multirow[b]{2}{*}{ Projectile } & \multicolumn{2}{|c|}{$10 \mathrm{MeV}$} & \multicolumn{2}{|c|}{$100 \mathrm{MeV}$} & \multicolumn{2}{|c|}{$1 \mathrm{GeV}$} \\
\hline & $\sigma_{c}^{(2)}$ & $\sigma_{f l}^{(2)}(1)$ & $\sigma_{c}^{(2)}$ & $\sigma_{f l}^{(2)}(1)$ & $\sigma_{c}^{(2)}$ & $\sigma_{f l}^{(2)}(1)$ \\
\hline${ }^{40} \mathrm{Ca}$ & 1.01 & 4.52 & 2.17 & 2.19 & 7.20 & 0.72 \\
\hline${ }^{120} \mathrm{Sn}$ & 12.37 & 49.54 & 26.48 & 22.94 & 72.61 & 6.65 \\
\hline${ }^{132} \mathrm{Xe}$ & 15.03 & 59.62 & 32.19 & 27.57 & 88.50 & 8.00 \\
\hline${ }^{165} \mathrm{Ho}$ & 23.32 & 90.61 & 51.13 & 42.60 & 138.59 & 12.34 \\
\hline${ }^{208} \mathrm{~Pb}$ & 40.45 & 148.66 & 96.95 & 72.87 & 234.84 & 19.83 \\
\hline${ }^{238} \mathrm{U}$ & 47.05 & 174.78 & 109.15 & 84.86 & 276.53 & 24.04 \\
\hline
\end{tabular}

TABLE II. Contributions of the coherent and fluctuation components to the TGDR excitation cross section (in mb) of various projectiles incident on a lead target at several different values of the incident energy.

\begin{tabular}{|c|c|c|c|c|c|c|c|c|c|}
\hline \multirow[b]{2}{*}{ Projectile } & \multicolumn{3}{|c|}{$10 \mathrm{MeV}$} & \multicolumn{3}{|c|}{$100 \mathrm{MeV}$} & \multicolumn{3}{|c|}{$1 \mathrm{GeV}$} \\
\hline & $\sigma_{c}^{(3)}$ & $\sigma_{f l}^{(3)}(2)$ & $\sigma_{f l}^{(3)}(1)$ & $\sigma_{c}^{(3)}$ & $\sigma_{f l}^{(3)}(2)$ & $\sigma_{f l}^{(3)}(1)$ & $\sigma_{c}^{(3)}$ & $\sigma_{f l}^{(3)}(2)$ & $\sigma_{f l}^{(3)}(1)$ \\
\hline${ }^{40} \mathrm{Ca}$ & 0.01 & 0.08 & 0.11 & 0.02 & 0.06 & 0.02 & 0.11 & 0.02 & 0.00 \\
\hline${ }^{120} \mathrm{Sn}$ & 0.17 & 2.46 & 3.17 & 0.84 & 1.92 & 0.64 & 3.03 & 0.47 & 0.04 \\
\hline${ }^{132} \mathrm{Xe}$ & 0.23 & 3.20 & 4.10 & 1.10 & 2.50 & 0.83 & 4.07 & 0.62 & 0.05 \\
\hline${ }^{165} \mathrm{Ho}$ & 0.42 & 5.81 & 7.37 & 2.08 & 4.70 & 1.54 & 7.76 & 1.17 & 0.09 \\
\hline${ }^{208} \mathrm{~Pb}$ & 0.95 & 12.24 & 14.83 & 5.28 & 10.78 & 3.36 & 16.68 & 2.40 & 0.18 \\
\hline${ }^{238} \mathrm{U}$ & 1.18 & 15.09 & 18.60 & 6.13 & 13.14 & 4.22 & 21.01 & 3.14 & 0.24 \\
\hline
\end{tabular}

